\title{
Chichinmanum weamu*: bienestar de los estudiantes awajún en la Universidad Nacional de la Amazonía Peruana
}

\author{
Alejandra Hidalgo Bonicelli \\ Pontificia Universidad Católica del Perú \\ alejandra.hidalgo.b@gmail.com
}

RESUMEN

Chichinmanum weamu, traducido como yendo a contracorriente, describe una experiencia por la cual atraviesan muchos jóvenes indigenas universitarios al enfrentar situaciones que afectan su bienestar. Esta investigación analiza si las condiciones en las que estudiaban seis jóvenes awajún en la Universidad Nacional de la Amazonía Peruana garantizaban ese bienestar. Con este propósito, utilizamos una metodología cualitativa y diferentes técnicas etnográficas como entrevistas, grupos focales, observaciones e historias de vida. Argumentamos que estar bien significaba para los estudiantes awajún contar con todos los servicios básicos, bienes y recursos que incluyen relaciones cercanas con sus compañeros y docentes. Al observar dichos aspectos, concluimos que los estudiantes awajún no estaban satisfechos. Sin embargo, el capital social desarrollado a través de la Organización de Estudiantes de los Pueblos Indígenas de la Amazonía Peruana les garantizaba su sobrevivencia en Iquitos.

Palabras clave: educación superior, juventud indigena, awajún, migrantes, bienestar.

\footnotetext{
Significa «yendo a contracorriente» en awajún.
} 


\title{
Chichinmanum weamu**: awajun in the National University of the Peruvian Amazon
}

\author{
SUMMARY
}

This article analyzes if life conditions in the National University of the Peruvian Amazon assured six young Awajun their well-being. It ponders several questions; what is the emic meaning of being well?, Could young migrants satisfy their socio-economic and affective needs?, which are the strategies used to perform as students? To answer these questions, a qualitative methodology and different ethnographic techniques such as interviews, focus groups, observations and life stories were used. And different actors such as student colleagues, teachers, university authorities, were observed and interviewed.

The expression chichinmanum weamu, translated from Awajún as going against the current, describes a situation that many young natives who try to become graduates go through. Young Awajun thought that being good meant having all the basic services, goods and resources needed to develop as students, which also implied having fluid relationships with peers and teachers. The study reached the conclusion that young Awajun were not entirely satisfied. However, the social capital developed through the Student Organization of the Indigenous Peoples of the Peruvian Amazon did ensure their survival in Iquitos.

Keywords: higher education, indigenous youth, Awajun, migrants, well-being.

** $\quad$ Awajun meaning for going against the current. 


\section{INTRODUCCIÓN}

El tema de la investigación se enmarca dentro de los estudios sobre educación superior, que en el Perú se caracteriza por tener un acceso desigual, en donde los pueblos amazónicos son los menos propensos a terminar la secundaria y matricularse en la universidad (Castro y Yamada, 2011). De acuerdo con la Encuesta Nacional de Hogares (Enaho) de 2016, un 31\% de la población mayor a los 18 años accede a educación superior y solo el $12 \%$ de ese total tiene una lengua materna indígena (INEI, 2016). A pesar de que la regulación aplicable lo exija, la mayoría de universidades no muestra interés por incorporar un enfoque intercultural en su enseñanza ${ }^{1}$. Entre otras razones, esto se debe a que en el Perú no existe un consenso sobre lo que implica ser una institución intercultural, lo cual es necesario para diseñar e implementar programas de acción afirmativa efectivos (Sanborn y Arrieta, 2012). La despreocupación de las instituciones en las filiaciones étnicas de sus estudiantes se interpreta como una falta de interés en su identidad, lo cual afecta directamente su bienestar.

Por otro lado, los estudios sobre el bienestar se caracterizan por ser interdisciplinarios, ya que las investigaciones provenientes de las ciencias sociales se complementan con estudios psicológicos. Desde la psicología social se señala que, si bien se han desarrollado investigaciones sobre el bienestar y necesidades en diferentes lugares de la región amazónica, no hay investigaciones de carácter emic que integren distintas percepciones del bienestar provenientes de variadas

\footnotetext{
1 El artículo 20 de la Ley General de Educación indica que «la educación (bilingüe) intercultural se ofrece en todo el sistema educativo» (Espinosa de Rivero, 2008).
} 
zonas de la Amazonía (Pons, 2013). Esta investigación plantea contribuir a ese vacío de conocimiento, ya que el objetivo principal fue analizar las vivencias de seis jóvenes estudiantes awajún que se encuentran en un nuevo contexto — es decir, en la Universidad Nacional de la Amazonía Peruana (UNAP) — e identificar si dichas condiciones les garantizan bienestar. La etapa universitaria puede resultar difícil para muchos jóvenes, ya que implica una transición llena de cambios. Sin embargo, las dificultades se intensifican para los jóvenes indígenas, quienes han migrado de distintas comunidades nativas a una ciudad como Iquitos. A pesar de que la investigación se guio por el concepto emic de bienestar, la definición etic - es decir, la función del grado de congruencia entre los deseos, aspiraciones, y necesidades individuales por un lado, y las oportunidades y demandas del medio ambiente por el otro - encaminó la metodología (Omar, 2006)².

La primera sección de este artículo muestra un estado de la cuestión sobre la educación superior indígena que incluye algunos avances y desafíos pendientes. La segunda, resume las implicancias de bienestar de los jóvenes awajún, y la tercera aborda el tema de satisfacción de necesidades socioeconómicas y afectivas. Luego, la cuarta muestra estrategias que desarrollan los estudiantes para enfrentar distintos desafíos. Finalmente, concluimos con unas reflexiones acerca de cómo incrementar el bienestar de los jóvenes indígenas en contextos universitarios, específicamente el de los awajún en la UNAP.

\section{EDUCACIÓN SUPERIOR INDÍGENA}

En América Latina se han hecho esfuerzos por crear instituciones de educación superior con enfoques novedosos. Por un lado, se encuentran las universidades indígenas destinadas explícitamente a poblaciones indígenas y basadas en el enfoque anglosajón, que busca empoderar a las minorías. Dichas instituciones son llamadas paralelas, ya que que son administradas por los pueblos indígenas y tienen como ventaja la falta de discriminación (Sarfaty, 2011). Entre sus desventajas se encuentra la posibilidad de ser un gueto académico (Cuenca, 2012). Un ejemplo es el programa de Formación de Maestros Bilingües de la Amazonía Peruana (Formabiap), creado por la Asociación Interétnica de Desarrollo de la Selva Peruana (Aidesep).

2 La definición emic proviene de los mismos estudiantes awajún, mientras que la definición etic proviene de las distintas disciplinas. 
Por otro lado, encontramos las universidades interculturales que se dirigen a toda la sociedad y mantienen un principio de «interculturalidad para todos», ya que se basan en el enfoque europeo continental que busca transversalizar las competencias interculturales entre las minorías marginadas y las mayorías marginadoras (Dietz, 2009). En el Perú se creó la Universidad Nacional Intercultural de la Amazonía (UNIA) en 1999 como producto de la reivindicación de los pueblos amazónicos liderados bajo Aidesep. Sin embargo, esta institución no se caracteriza por ser intercultural en la práctica, ya que la experiencia de los jóvenes indígenas amazónicos en muchos casos está marcada por el racismo (Sarfaty, 2011).

A mediados de la década de 1980, grupos de jóvenes indígenas amazónicos migraron a distintas ciudades con el fin de acceder a la educación superior, progresar y alcanzar el bienestar (Espinosa de Rivero, 2012) ${ }^{3}$. Desde comienzos de la década de 1990, el acceso de los pueblos indígenas peruanos a la educación superior fue posible gracias a la existencia de mecanismos y políticas conocidas como acciones afirmativas que tienen como objetivo asegurar la igualdad de oportunidades de grupos históricamente excluidos (Cunningham, 2008). Entre estas se encuentran las becas, cupos y convenios.

Este estudio entiende por interculturalidad al «enfoque que analiza y visibiliza las interacciones a menudo desiguales y asimétricas entre miembros de grupos cultural e identitariamente diversos (frente a la multiculturalidad, que solo contrasta la existencia de diferencias, sin estudiar sus interacciones e hibridaciones)» (Dietz, 2009, p. 56). Por otro lado, el interculturalismo es una propuesta político-pedagógica que considera estas interacciones como deseables (Dietz, 2009, p. 56). Asimismo, la interculturalidad piensa que la relación entre personas y grupos diversos está basada en el respeto y se percibe desde posiciones de igualdad (Schmelkes, 2004).

La experiencia de la Universidad Veracruzana Intercultural (UVI) en México, la cual se guía por el enfoque transversalizador, pone en evidencia que la interculturalidad no solo debe centrarse en las prácticas culturales pedagógicas que responden a lógicas culturales distintas, sino que también se deben tener en cuenta las interacciones que generan la diversidad etnolingüística y la multiplicidad de actores que se pueden ver envueltos en un contexto culturalmente diverso. Dichos actores incluyen a activistas, líderes, sabios locales y académicos, quienes

La motivación de acceder a instituciones educativas suele estar relacionada con el mito del progreso postulado por Carlos Iván Degregori, en donde el progreso y el bienestar son frutos de una adecuada educación (Degregori, 1986). 
son propietarios de distintos saberes (Dietz, 2009). Se señala la existencia de dos tipos de saberes, entre los cuales se encuentra el conocimiento científico presente en las universidades convencionales y cuya validez se asume implícitamente como universal. Por otro lado, existen conocimientos étnicos, populares o locales, los cuales en algunos contextos son considerados como particulares. La descalificación del segundo tipo de saberes surge como consecuencia de la herencia colonial. Frente a esto la interculturalidad propone un diálogo entre ambos tipos de saberes (Mato, 2007). La UVI desafia el «carácter aún universalista, monológico y "monoepistémico" de la universidad occidental clásica» (Dietz, 2009, p. 71) al incluir diversos actores y saberes regionales.

Con respecto a la lengua enseñada, es importante señalar que una educación bilingüe no necesariamente es una educación intercultural, ya que con el bilingüismo inicia el reconocimiento del otro, pero no necesariamente significa su aceptación. Algunos programas de Educación Intercultural Bilingüe (EIB) en lugar de tener un enfoque más general, se orientan hacia lo lingüístico, lo cual implica que los alumnos traduzcan, memoricen y terminen asimilando el sistema de educación formal, sacrificando procesos educativos tradicionales (Cunningham, 2008) $)^{4}$.

Entre los casos de acciones afirmativas que muestran inconvenientes al ser recibidas por los pueblos indígenas peruanos, se encuentra la incorporación de estudiantes amazónicos a la Universidad Nacional Mayor de San Marcos (UNMSM) y a la Universidad Nacional de San Antonio Abad del Cusco (UNSAAC). En ambos casos, el rendimiento académico no fue tomado en cuenta al dejar que las organizaciones indígenas eligieran a los beneficiarios y esto generó problemas como la deserción y desaprobación de cursos (Sanborn y Arrieta, 2012). Por otro lado, la investigación de Buu-Sao indica que en 2002 se firmó un acuerdo entre la Aidesep y la UNAP, en donde se crearon normas para la entrada de jóvenes indígenas provenientes de contextos de pobreza (Buu-Sao, 2011).

4 En América Latina existen importantes lecciones aprendidas de EIB para la interculturalidad. A pesar de esto, no hay un intercambio de experiencias entre países (Cunningham, 2008). En el Perú, Formabiap enseña la especialidad de educación primaria intercultural bilingüe y ha trabajado en comunidades indígenas amazónicas de Loreto, Amazonas, Ucayali, Junín y Pasco, elaborando diversos currículos para la educación primaria y materiales educativos en nueve lenguas indígenas. Entre las buenas prácticas se señala una modalidad mixta de ciclos presenciales en Iquitos y no presenciales en las comunidades de los alumnos para evitar el desarraigo de estas. Sus retos incluyen la dificultad para expandir la EIB en distintas realidades indígenas y la inserción laboral de los profesores (Trapnell, 2008). 
La mayor parte de instituciones que tienen acciones afirmativas, como los casos citados, suelen ofrecer únicamente admisión preferencial, y una vez que los estudiantes indígenas o de grupos étnicos ingresan a las instituciones, no se les da ayuda financiera directa y casi no se ofrecen programas propedéuticos o de acompañamiento académico (Sanborn y Arrieta, 2012). Al migrar a un contexto urbano con el fin de estudiar, los jóvenes indígenas se encuentran con desafíos económicos, académicos y socioculturales que generan un choque cultural (Espinosa de Rivero, 2007). Asimismo, se deja de lado el trabajo con estudiantes no indígenas para trabajar el problema de discriminación, es decir que no lo tratan como un proceso de doble vía y la acción afirmativa termina siendo un mecanismo multicultural y no intercultural (Cuenca, 2012).

La mayoría de jóvenes indígenas tienen que vivir en ciudades lejanas a sus comunidades de origen y necesitan medios económicos para gastos de manutención y materiales para la universidad. El estudio de Buu-Sao en Iquitos indica que las dificultades económicas son las que terminan provocando casos de deserción entre los jóvenes indígenas.

En algunas universidades casi no hay iniciativas que promuevan la filiación étnica o cultural de los estudiantes y que faciliten su adaptación. Buu-Sao señala que la falta de expresión oral de los estudiantes indígenas en Iquitos es frecuente, ya que su lengua materna no es el castellano. Como consecuencia, su autoestima disminuye, generando sentimientos de inferioridad y timidez frente a sus compañeros. Muchos jóvenes manifiestan un temor constante a ser discriminados, lo que en muchos casos los lleva a negar sus orígenes como indígenas, a distanciarse de la realidad amazónica y a cuestionar su propia identidad cultural. El desarraigo cultural suele ir acompañado del racismo de las ciudades y «todos estos elementos condicionan el "desarrollo psico-emocional" de los jóvenes indígenas» (Espinosa de Rivero, 2007, p. 15).

Entre los problemas de las universidades convencionales, se observa que los perfiles profesionales no ofrecen campos laborales que concuerden con las necesidades de los jóvenes indígenas que quieren regresar a sus pueblos, sino que promueven la migración hacia contextos urbanos (Dietz, 2009). En la UVI se responde a esta problemática buscando que los alumnos se vinculen con su comunidad a través de diferentes actividades como la gestión de proyectos y la investigación-acción en sus comunidades (Dietz, 2009).

Los pueblos indígenas plantean la importancia de que la educación responda a la preservación, fomento y desarrollo de las culturas ancestrales y que los mismos pueblos sean quienes diseñen, implementen y ejecuten las políticas educativas 
(Cunningham, 2008). Por lo tanto, la oferta de educación indígena debería contar con un modelo de gestión participativo y descentralizado (Cunningham, 2008).

Lamentablemente, la interculturalidad en la educación superior no ha sido una prioridad constante para los movimientos étnicos en Perú, a diferencia de otros países con población indígena. Las organizaciones peruanas basadas en identidades étnicas o raciales han sido históricamente reprimidas y débiles. En los últimos años, se ha generado una movilización política de los pueblos nativos de la Amazonía como los awajún y comunidades andinas que se han visto afectadas por las industrias extractivas. Sin embargo, se han enfatizado temas como la gestión de tierras y recursos naturales (Sanborn y Arrieta, 2012) .

\section{METODOLOGÍA}

Se realizó trabajo de campo entre los meses de marzo y mayo del 2014 y se utilizó una metodología cualitativa centrada en la etnografía y en estudios de caso sobre las vivencias de seis jóvenes estudiantes awajún de la UNAP.

Los criterios para elegir los casos fueron la variedad de carreras, el ciclo que cursaban y el tiempo que tenían viviendo en Iquitos. Las edades de estos jóvenes oscilaban entre los 18 y 30 años y el tiempo que tenían viviendo en Iquitos variaba de nueve años a meses, ya que los ciclos en que estaban inscritos iban desde el primero hasta el noveno. La mayoría provenía de comunidades localizadas en la provincia de Datém del Marañón, en Loreto como Atahualpa y Ajachim, excepto por dos estudiantes que provenían de la provincia de Condorcanqui en Amazonas. Se trabajó con dos chicas y cuatro chicos, ya que el grupo de jóvenes awajún era mayoritariamente masculino.

Los instrumentos metodológicos utilizados se adecuaron al contexto e informantes respectivos. La herramienta más utilizada fue la entrevista semiestructurada, seguida por la observación y los grupos focales. Se entrevistó a los jóvenes universitarios awajún acerca de las motivaciones que impulsan la migración, la relación entre la educación y sus aspiraciones, la satisfacción de necesidades socioeconómicas y afectivas, las oportunidades que se tienen con respecto al servicio educativo y los retos encontrados en la UNAP y en Iquitos.

5 El «baguazo» es un evento violento que mostró la crisis política que se vivía al interior del país durante el segundo gobierno de Alan García en 2009. Los pueblos indígenas protestaron unidos a través de la Aidesep en contra de la empresa petrolera estatal Petroperú, lo cual causó muchas muertes siendo uno de los sucesos de violencia política más recordados en la última década. Véase Cavero (2011). 
También se utilizaron entrevistas no estructuradas para averiguar acerca de las posibles estrategias estudiantiles para sobrellevar la vida universitaria. Se eligió esta técnica para abordar a distintos actores, entre los que se encuentran los docentes y el personal administrativo de la UNAP, principalmente los trabajadores de la Oficina General de Bienestar Universitario (Ogebu); instituciones indígenas entre las que se encuentran la Aidesep y la Organización de Estudiantes de los Pueblos Indígenas de la Amazonía Peruana (Oepiap); la Red Ambiental Loretana (RAL); el Gobierno Regional de Loreto (Gorel), y finalmente, los amigos de los jóvenes awajún.

Los nombres de todos los participantes de esta investigación se muestran con sus iniciales con el fin de mantener la confidencialidad. Las iniciales de los seis jóvenes en los cuales se centró la investigación son J.M.A., G.M.T., D.C.P., A.U.J., Z.C.A. y F.M.K., y se diferencian de las iniciales de los demás actores por tener tres letras.

\section{IMPLICANCIAS DE ESTAR BIENY RELEVANCIA DE ESTAS PARA UN JOVEN UNIVERSITARIO AWAJÚN}

Para conocer si los jóvenes se encontraban satisfechos con las condiciones del contexto urbano fue necesario saber qué es lo que los jóvenes universitarios awajún consideraban como estar bien. Comparar la concepción de estar bien de los estudiantes no indígenas de la UNAP con la de los jóvenes awajún hizo más notorio los factores relevantes para los jóvenes indígenas.

La categoría emic de estar bien se construyó a partir del reconocimiento de los factores que los jóvenes awajún consideran necesarios para alcanzar el bienestar, la relevancia que cada individuo da a cada factor y mediante la identificación de los significados personales. A pesar de que se ha buscado obtener una definición emic de bienestar, la metodología se ha planteado sobre la base del concepto etic, por lo que se consideró importante preguntar a los estudiantes qué pensaban de los componentes que no se observaba en sus respuestas sobre las implicancias de bienestar. En la presente investigación, la definición etic de bienestar es la función del grado de congruencia entre los deseos o las aspiraciones, y necesidades individuales, por un lado, y las oportunidades y demandas del medio ambiente por el otro (Omar, 2006). Por lo tanto, los principales componentes del concepto etic son las aspiraciones, necesidades, oportunidades y demandas. Se habló del tema en un grupo de tres y en otro de dos y en el caso de Z.C.A. de manera personal. 
Entre las implicancias de estar bien de los estudiantes awajún, se encuentran la satisfacción de diferentes necesidades y hacer frente a los retos con los que se encuentran. En un primer momento no se habló acerca de las aspiraciones personales. Sin embargo, cuando se pregunta a los jóvenes por la importancia de este componente para estar bien, algunos le dan más importancia que otros, aunque lo conciben como explícito y lo relacionan con el deseo de ser profesional. Se señala que el bienestar es contar con las necesidades básicas como la alimentación, el alojamiento y los materiales para desempeñar su rol. Las implicancias de estar bien tienen distinta prioridad, en donde los factores más importantes se posicionan arriba:

Tabla 1. Orden de factores para los estudiantes awajún* (2017)

\begin{tabular}{|c|c|c|c|c|}
\hline J.M.A. & G.M.T. & Z.C.A. & D.C.P. & F.M.K. \\
\hline $\begin{array}{l}\text { Bienes } \\
\text { materiales } \\
\text { y acceso a } \\
\text { servicios }\end{array}$ & Salud & $\begin{array}{l}\text { Bienes } \\
\text { materiales } \\
\text { y acceso a } \\
\text { servicios }\end{array}$ & Aspiraciones & Aspiraciones \\
\hline $\begin{array}{l}\text { Relaciones } \\
\text { sociales } \\
\text { (amistades) }\end{array}$ & $\begin{array}{l}\text { Bienes } \\
\text { materiales } \\
\text { y acceso a } \\
\text { servicios }\end{array}$ & $\begin{array}{l}\text { Relaciones } \\
\text { sociales } \\
\text { (amistades) }\end{array}$ & Salud & Salud \\
\hline $\begin{array}{l}\text { Comodidad del } \\
\text { lugar donde se } \\
\text { vive }\end{array}$ & $\begin{array}{l}\text { Relaciones } \\
\text { sociales } \\
\text { (amistades) }\end{array}$ & & $\begin{array}{l}\text { Relaciones } \\
\text { sociales } \\
\text { (amistades) }\end{array}$ & $\begin{array}{l}\text { Relaciones } \\
\text { sociales } \\
\text { (amistades) }\end{array}$ \\
\hline Aspiraciones & Aspiraciones & & $\begin{array}{l}\text { Bienes } \\
\text { materiales } \\
\text { y acceso a } \\
\text { servicios }\end{array}$ & $\begin{array}{l}\text { Bienes } \\
\text { materiales } \\
\text { y acceso a } \\
\text { servicios }\end{array}$ \\
\hline
\end{tabular}

* A.U.J. no estuvo presente durante esta parte del grupo focal.

Comparación entre la concepción de estar bien para un joven universitario awajún y la concepción de estar bien para un estudiante no indígena de la UNAP

Esta comparación permitió conocer las particularidades del caso a estudiar, ya que la etapa universitaria afecta muchos aspectos independientemente de la cultura a la cual pertenezca un individuo. La comparación permite reconocer las diferencias 
entre ambos significados y el significado particular del concepto de estar bien para los jóvenes awajún. Los estudiantes no indígenas con los que se trabajó son amigos y compañeros de carrera de algunos de los estudiantes awajún: M.F., E.S. y F.V. A diferencia de los estudiantes awajún, los estudiantes no indígenas no recalcan la importancia de la satisfacción de necesidades y servicios básicos como la alimentación y la vivienda, ya que todos vivían con familiares.

Las implicancias de estar bien en las que coincidían los estudiantes no indígenas entrevistados fueron estar en constante contacto tanto con los familiares como con los amigos y tener buena salud. F.V. consideraba que estar bien como estudiante es sentirse capaz de ser académicamente mejor que otras personas porque considera que es importante ser competitivo. M.F. indicó que los estudios son importantes porque se relacionan directamente con el trabajo y la economía en el futuro, por consiguiente, considera que el significado de bienestar para un estudiante es principalmente tener buenas notas en los cursos.

Al igual que en el caso de los jóvenes estudiantes awajún, no se señala a las aspiraciones como un factor explícito necesario para estar bien, aunque las consideran importantes:

Tabla 2. Orden de factores para los estudiantes no indígenas (2017)

\begin{tabular}{lll}
\hline M. F. & F. V. & E. S. \\
\hline Aspiraciones & Aspiraciones & Relaciones familiares \\
Cercanía con la familia & Salud & Amistades \\
Salud & $\begin{array}{l}\text { Relaciones sociales } \\
\text { (familiares y amistades) }\end{array}$ & Aspiraciones \\
Estudios & $\begin{array}{l}\text { Bienes materiales y acceso } \\
\text { a servicios }\end{array}$ & $\begin{array}{l}\text { Bienes materiales y acceso } \\
\text { a servicios }\end{array}$ \\
\hline
\end{tabular}

En conclusión, los jóvenes universitarios awajún consideran que estar bien es acceder a las necesidades básicas, mantener buenas relaciones y acceder a bienes que les permitan cumplir con su rol de estudiantes y a su vez formarse como profesionales con diferentes conocimientos y habilidades. En cambio, los jóvenes no indígenas le dan menos importancia al acceso de bienes y servicios que a las relaciones sociales. 


\section{SATISFACCIÓN DENECESIDADES SOCIOECONÓMICASYAFECTIVAS}

La mayoría de estudiantes indígenas de la UNAP pertenece a la Oepiap, la cual también está integrada por estudiantes que asisten a otros centros educativos. Los estudiantes indígenas pertenecen a los pueblos que habitan en la región del Alto Amazonas, como los awajún, achuar, kichwas, shawi, shiwilo y wampis, y a los pueblos de la región de Bajo Amazonas, como los bora, huitoto, kichwa, kukama, matsés y tikuna.

\section{Gráfico 1. Distribución de la Oepiap por pueblos indígenas}

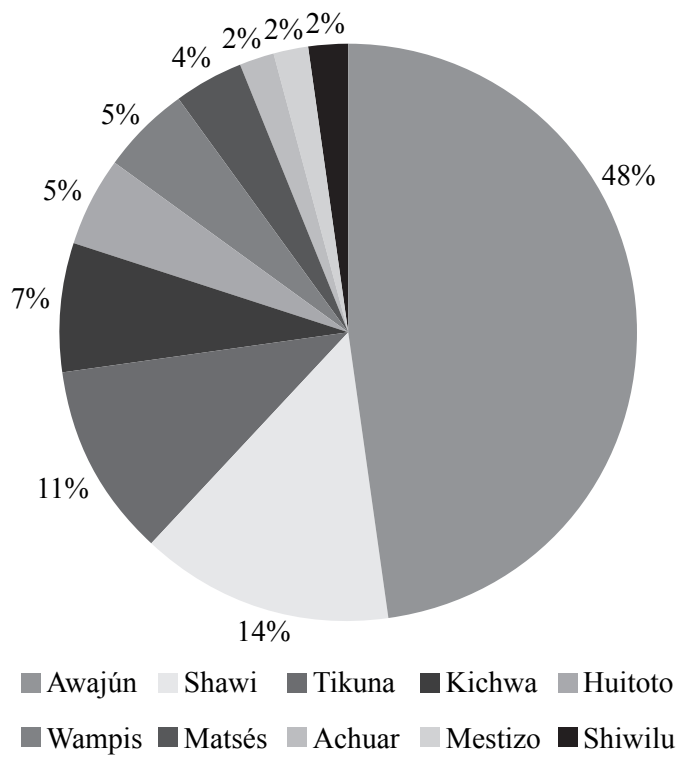

Elaboración propia a partir de la lista de integrantes de la Oepiap de la revista Waymaku de mayo de 2011.

Los jóvenes coincidían en que vivir en la ciudad es un proceso difícil. La Oepiap y el Gorel han aliviado algunas de las necesidades socioeconómicas de los estudiantes indígenas. Sin embargo, las primeras generaciones de estudiantes indígenas tenían retos como darse a conocer frente a las autoridades universitarias y visibilizarse, reclamar por la matrícula gratuita y el acceso al comedor universitario que se les debía otorgar, ya que era parte de la Ley Universitaria creada por Alberto Fujimori.

J.M.A. mencionó cinco puntos que contribuyen al choque cultural provocado por la migración: (i) la incapacidad de poder expresarse adecuadamente en 
castellano, (ii) la nostalgia por las costumbres de su pueblo, (iii) la diferencia entre las viviendas urbanas y las rurales, (iv) la marginación por parte de sus compañeros no indígenas, y finalmente, (v) la desventaja educativa. Todos estos problemas o retos son compartidos por la mayor parte de jóvenes indígenas.

Además, la ciudad tiene muchas distracciones como las películas, los partidos de fútbol, las bebidas alcohólicas; elementos con los que no solían relacionarse en sus comunidades y que en la ciudad forman parte de la vida social de los iquiteños

Asimismo, los jóvenes que provienen de comunidades indígenas llegan a Iquitos con muy pocos recursos económicos, y una vez que están viviendo en la ciudad, no cuentan con un apoyo económico considerable y estable de sus familias, ya que la mayoría tiene una economía de autoconsumo. Algunos chicos tienen padres que se dedican a la docencia y que poseen un poco más de recursos, aunque estos continúan siendo limitados.

Los estudiantes awajún necesitaban materiales, especialmente libros y fotocopias. Además, el uso de la computadora resulta fundamental en las carreras relacionadas con ciencias y en las que se realizan prácticas de laboratorio como Ingeniería Química e Ingeniería y Gestión Ambiental.

\section{Acceso a servicios}

Los estudiantes awajún vivían en el local de la Organización Regional de los Pueblos Indígenas del Oriente (Orpio), la RAL, sede principal de la Oepiap, y el Hospedaje Nissi. El local de la Orpio es el primero en el cual los estudiantes indígenas organizados como la Oepiap comenzaron a vivir desde 2002. En dicho local se encuentran las oficinas de diversas federaciones, organizaciones regionales y las de Formabiap. A.U.J. señaló que parte de las paredes de triplay están rotas, ahí vivía con veinte estudiantes indígenas, de los cuales siete eran estudiantes awajún y todos debían pagar por el servicio del agua.

Por otro lado, en 2009 la Oepiap construyó un albergue estudiantil de diez malocas ${ }^{6}$ con la ayuda del Gorel en el local de la RAL, terreno que pertenece a la Iglesia católica. Se hizo un acuerdo con P.M. que establecía el alojamiento de los estudiantes durante cinco años, que caducó en 2014, y los estudiantes tuvieron que conseguir otro alojamiento. Aproximadamente 38 estudiantes indígenas vivían en la RAL, entre ellos, dieciocho awajún. G.M.T. señaló que se encontraba

\footnotetext{
6 Casa comunal tradicional de los pueblos indígenas de la Amazonía.
} 
cómoda porque era un espacio parecido a la comunidad, pero que los materiales estaban viejos. En ese caso pagaban S/. 10 mensuales por la luz.

Por último, se encuentra el hospedaje Nissi, el cual es una casa de dos pisos. Había un aproximado de veinticinco estudiantes indígenas hospedados: diez estudiaban en la UNAP y cinco eran awajún. Sin embargo, los estudiantes se sentían incómodos por el constante retraso de pagos del Gorel, lo cual les traía daños psicológicos, ya que les generaba inseguridad ${ }^{7}$.

Uno de los principales apoyos económicos que la UNAP brinda a los estudiantes gracias al Convenio tripartito con el Gorel y Aidesep es el acceso al comedor universitario. Dicho acuerdo señalaba que hay pensiones alimenticias para un cupo de cincuenta estudiantes indígenas (Aidesep-Gorel-UNAP, 2012, pp. 3 y 5). Por lo tanto, la mayoría de estudiantes awajún accede al comedor. Este no atiende ni domingos ni feriados, lo cual es una dificultad para los jóvenes indígenas. R.D., jefe de la Ogebu de la UNAP, indica que el 50\% de estudiantes que acceden al comedor son indígenas y que parte de las condiciones es que tengan un promedio aprobatorio, lo cual implica un reto para muchos estudiantes indígenas.

\section{Cercanía de la familia y amigos}

Algunos hermanos mayores que viven en Iquitos suelen traer a sus hermanos menores o primos para que puedan estudiar y tener las mismas oportunidades que ellos. La migración ha implicado alejarse de familiares y amigos del pueblo. A pesar de esto, la mayoría de los seis jóvenes vivían con algunos primos y hermanos $^{8}$. Asimismo, muchos jóvenes forman su propia familia cuando están en la ciudad, y es interesante notar que una característica de los líderes de la Oepiap ha sido ser padres de familia, como es el caso de J.M.A.

Con respecto a las amistades, al llegar a la ciudad los jóvenes se rodean de compañeros que son diferentes en aspectos culturales y económicos, por lo cual

7 En 2015, cuando se realizó la devolución del trabajo de campo, los seis estudiantes awajún vivían en diferentes lugares. La Oepiap firmó un convenio para que el actual Gorel pague el alojamiento de algunos estudiantes indígenas. J.M.A. y F.M.K. vivían en un hospedaje de la cuadra 5 de la calle Ricardo Palma; A.U.J. y D.C.P. vivían en otro hospedaje de la cuadra 4 de la calle Sargento Lores, G.M.T. vivía en la casa de la madre de su pareja y Z.C.A. retornó a su pueblo. La Oepiap estaba intentando que el Gorel construya el albergue estudiantil pendiente y que exista un compromiso legal.

8 J.M.A. y G.M.T. son primos y gran parte de su familia estaba viviendo en Iquitos, ya que los hermanos de J.M.A. migraron anteriormente. Z.C.A. vivía con su hermano, D.C.P. y F.M.K. tenían primos y la hermana menor de A.U.J. estaba internada en un colegio de Iquitos. 
los jóvenes indígenas suelen agruparse, ya que es más complicado hacer amigos cuando no se domina perfectamente el castellano. La facilidad para hacer amigos depende de la personalidad de cada uno y de las características de las facultades de los estudiantes, ya que hacer amigos es mucho más factible en una facultad pequeña en donde hay más confianza, incluso con los profesores, y donde las personas son más acogedoras. J.M.A. señaló que sus compañeros de Antropología son más humanitarios que los de Administración, carrera en la cual se sentía discriminado y marginado. Se asistió a un par de reuniones organizadas por la Asamblea de Alumnos de Antropología, en donde J.M.A. participó bastante y era considerado por algunos de sus compañeros como un líder. El mejor amigo de Antropología de J.M.A. es M. F., quien es de Tarapoto e indicó que durante el comienzo del primer año en Antropología los estudiantes indígenas se sentaban en un lado del salón y los mestizos ${ }^{9}$ se sentaban en la otra mitad del salón. Después de una semana, se comenzaron a apoyar en clases porque los estudiantes siempre querían hacer grupo con los chicos indígenas por sus diferentes habilidades en las prácticas de campo y cursos de supervivencia.

En la UNAP no hay ningún tipo de acogida para que los jóvenes indígenas presenten su cultura, lo cual sería un buen modo de promover la interculturalidad en la universidad. En ocasiones, los jóvenes indígenas se mimetizan con el resto de alumnos y niegan su propia identidad (Oepiap, 2011, p. 15). Sin embargo, no se observó eso en ninguno de los casos.

Al hablar con los amigos no indígenas de los jóvenes awajún, concuerdan en que al comienzo no se daban cuenta de que los jóvenes awajún provenían de una comunidad indígena y ellos mismos se excluían. Los amigos y los profesores indican que la mayor parte de los jóvenes awajún suele ser de perfil bajo en el salón de clases, lo cual no necesariamente quiere decir que su desempeño sea malo, pero la falta de participación en clases es una característica en común.

Algunos profesores opinan que muchos chicos suelen ser muy tímidos en la universidad. Sin embargo, algunos son extrovertidos e incluso líderes al interior de sus federaciones u organizaciones indígenas como la Oepiap. P.M. señaló que, en ocasiones, algunos estudiantes indígenas no muestran su lado indígena al interior de las aulas, pero cuando lo hacen, algunos ganan amigos por la apertura y la honestidad. También indicó que solía haber prejuicios entre los diferentes grupos indígenas que vivían en la RAL.

Muchos entrevistados utilizaban este término para referirse a las personas que no pertenecen a los pueblos amazónicos. 


\section{Retos académicos}

Además de preguntar a los estudiantes cuáles consideraban que eran sus principales retos académicos, fue importante conocer la percepción de sus profesores y compañeros. Los profesores consideraban que el rendimiento de sus alumnos indígenas era de regular abajo. Por otra parte, indicaron que tienen diferentes habilidades prácticas y que aprenden haciendo. Los docentes entrevistados comparten la idea de que las dificultades académicas de los estudiantes indígenas se relacionan con la falta del manejo del castellano y dificultades en las matemáticas y ciencias.

F.M.K. no había recibido ninguna evaluación corregida cuando fue entrevistado y aún no sabía cuáles eran sus mayores dificultades académicas. Sin embargo, mencionó que le costaba seguir las clases porque eran muy rápidas. De igual manera, Z.C.A. señaló que los profesores explican muy rápido y no les suele preguntar después de clase, ya que seguramente le dirían «esto es la universidad, no el colegio»».

D.C.P. y A.U.J. estudiaban ingeniería y gestión ambiental, Z.C.A. economía y F.M.K. ingeniería química, las cuales son carreras desafiantes por implicar un mayor uso de las ciencias y matemáticas. Muchos docentes opinaban que los estudiantes indígenas tienen menores dificultades académicas en cursos de sociales o letras que en cursos de ciencias, por lo que algunos señalan que es más conveniente que sigan carreras como Derecho ${ }^{10}$.

A.U.J. señaló que no tener recursos económicos para realizar investigaciones en cabinas de internet era un reto académico, ya que perdía notas de prácticas al no poder investigar o realizar informes. Su compañera R. indicó que A.U.J. jaló el curso por segunda vez por no saber utilizar la computadora ni mandar correos electrónicos.

R.T., profesor en agronomía, mencionó que el bajo desempeño de los estudiantes indígenas es causado porque desarrollan pocas capacidades al momento de ingresar a la universidad. Si bien a través de la modalidad de ingreso de pueblos indígenas los estudiantes compiten por las plazas, el esfuerzo que realizan no se compara con el examen de admisión, el cual impulsaría exigencias ${ }^{11}$.

10 Un ejemplo de esto es E.K., quien es awajún y logró graduarse de la especialidad de Derecho.

11 El objetivo principal de la modalidad de pueblos indígenas es demostrar que los jóvenes indígenas provienen del pueblo que figura en el certificado que han mostrado y que es acreditado por sus comunidades. Los postulantes tienen que demostrar que conocen el idioma y las tradiciones a través de relatos ancestrales. Se toman en cuenta las notas que los estudiantes han 
M.S., asesora pedagógica en el área de educación de la Aidesep, señaló que los alumnos indígenas arrastran el déficit de la educación que han recibido en sus comunidades durante la primaria y secundaria, ya que hay una falta de preparación intercultural en los profesores. Ella indicó la importancia de un curso propedéutico que se encargue de nivelar y fortalecer el rendimiento de los estudiantes indígenas en áreas básicas. Sin embargo, resaltó que no se pueden dejar de fortalecer los conocimientos indígenas para lograr una nivelación adecuada.

S.T. señaló que el rendimiento académico de los jóvenes indígenas mejora cada año en la UNAP y que cada vez hay menos desaprobados. Asimismo, P.M. indicó que la mejora del rendimiento académico ha sido un proceso de evolución, ya que hasta hace un par de años el rendimiento era muy malo y muchos estudiantes indígenas perdieron cupo en el comedor. Señaló que actualmente los estudiantes indígenas están casi a la par de los mestizos.

Si bien los jóvenes acceden a los servicios básicos con la ayuda de la Oepiap, la RAL y el Gorel, la mayoría de veces los recursos económicos son insuficientes para realizar investigaciones o trabajos vitales para aprobar los cursos. Con respecto a las diferencias entre jóvenes no indígenas de bajos recursos e indígenas, los estudiantes no indígenas tienen menos problemas de adaptación y socialización, en especial si provienen de contextos urbanos.

\section{ESTRATEGIAS PARA DESEMPEÑAR EL ROL DE UNIVERSITARIOS}

Los estudiantes awajún acceden a servicios básicos de alimentación y vivienda y mantienen relaciones con el Gorel a través de la Oepiap, tienen contactos con diferentes asociaciones de la Aidesep y algunos mantienen buenas relaciones con sus profesores y compañeros. En los últimos tiempos, la mayor parte de estudiantes aprueba sus cursos, a pesar de que conseguir materiales y recursos económicos sea difícil en ocasiones. El hecho de que se estén adaptando al contexto universitario indica que tienen ciertas estrategias económicas, de socialización y académicas, las cuales suelen articularse.

Además de dichas estrategias, llegar a Iquitos implicó un apoyo de sus familiares, ya que los ayudaron o informaron acerca de la facilidad que tenían para ingresar a la UNAP a través de la modalidad de pueblos indígenas. Los familiares

tenido durante el período escolar y de secundaria, pero se los exonera del examen de ingreso general, por lo cual personas no indígenas tratan de entrar por medio de esta modalidad. 
pueden proponer a los estudiantes estudiar en Iquitos. Sin embargo, en la mayoría casos ellos tienen que trabajar en sus comunidades para pagar su pasaje.

Al preguntar a los estudiantes awajún acerca de las mayores dificultades que tuvieron que pasar para adaptarse, J.M.A. y G.M.T. señalaron que tenían problemas con la comunicación y, por ende, con la socialización y el ámbito académico. Asimismo, F.M.K. y Z.C.A. indican que sus mayores dificultades también eran académicas. Por otro lado, las mayores dificultades para D.C.P. y A.U.J. eran económicas y por eso trabajaban en el verano o en alguna oportunidad en que les salga algún cachuelo ${ }^{12}$.

Los recursos para generar estrategias son el apoyo de familiares, compañeros universitarios, de la universidad, y específicamente de la Ogebu, de la RAL y de las organizaciones indígenas, principalmente de la Oepiap, quien es intermediaria entre los estudiantes indígenas y el Gorel.

La mayor parte de los estudiantes awajún sienten que la Oepiap es uno de los principales apoyos con el cual pueden contar y generar más influencias y contactos. J.M.A. fue el único de los seis estudiantes awajún que vivía en Iquitos cuando se creó la organización en 2003 con el fin de hacer frente a los problemas económicos, y fue presidente de la Oepiap hasta marzo de 2014 (Buu-Sao, 2011, p. 68). En mayo de 2006, los estudiantes indígenas agrupados en la Oepiap solicitaron el acceso al comedor universitario, la exoneración del pago de la matrícula, el ingreso a través de la modalidad de pueblos indígenas y el reforzamiento académico gratuito dentro de la CEPRE-UNAP a través de la toma del Rectorado. J.M.A. señala que lograron que se cumpliera la gratuidad del comedor universitario y el ingreso por la modalidad de pueblos indígenas. Con respecto al pago de la matrícula, solo les exoneraron un $50 \%$. Por otro lado, las autoridades de la UNAP se negaron a proveer reforzamiento académico a través de la CEPRE-UNAP y sustentaron que esa preparación debía darse durante los estudios secundarios. Lamentablemente, la educación formal rural que reciben los estudiantes indígenas se encuentra en desventaja con respecto a la educación recibida en Iquitos.

El Convenio de Cooperación Interinstitucional entre el Gorel, la UNAP y la Aidesep fue promovido por la Gerencia Regional de Asuntos Indígenas del Gorel y la creación de dicho organismo fue propuesto por W.M., hermano de J.M.A., quien fue elegido Consejero Regional de la provincia de Datém del Marañón en 2010 y permitía relaciones fluidas entre Oepiap y el Gorel.

12 Trabajo temporal e informal. 
La falta de recursos económicos es un factor que dificulta el desempeño académico de los estudiantes awajún. A diferencia de los problemas de socialización y las dificultades académicas que se pueden ir solucionando conforme el estudiante se va adentrando en la vida universitaria, los problemas económicos son enfrentados pidiendo el apoyo a diferentes instituciones a través de la Oepiap o a través de estrategias individuales.

Las estrategias económicas colectivas consisten en fortalecer el capital social a través de la Oepiap y utilizar la organización como una plataforma de conexión con otros actores sociales, como las autoridades de la UNAP, los municipios o el Gorel. El Gorel refaccionó los módulos de la RAL, brindó víveres, y en ocasiones colaboró en el pago de la luz. E.K., quien egresó de la Facultad de Derecho de la UNAP en el tiempo exacto que dura la carrera, señala que debe bastante a la Oepiap, porque gracias a esta organización pudo vivir en el local de la Orpio junto con sus hermanos y otros compañeros indígenas desde 2008 hasta que finalizó su carrera.

Con respecto a las estrategias económicas personales, los jóvenes tenían trabajos o cachuelos para poder comprar insumos y realizar las tareas universitarias:

Tabla 3. Trabajos de los jóvenes awajún (2014)

\begin{tabular}{|c|c|c|c|c|c|}
\hline & J.M.A. & G.M.T. & A.U.J. & D.C.P. & Z.C.A. \\
\hline $\begin{array}{l}\text { Tipo de } \\
\text { trabajo }\end{array}$ & $\begin{array}{l}\text { Asistente en la } \\
\text { Subgerencia de } \\
\text { Comunidades } \\
\text { Indígenas en el } \\
\text { Gorel }\end{array}$ & $\begin{array}{l}\text { Promotora de } \\
\text { ventas Avon- } \\
\text { Esika }\end{array}$ & $\begin{array}{l}\text { Trabajos de } \\
\text { construcción }\end{array}$ & $\begin{array}{l}\text { Recepcionista } \\
\text { en el hotel } \\
\text { Casa Morey }\end{array}$ & Niñera \\
\hline $\begin{array}{l}\text { Duración } \\
\text { del trabajo }\end{array}$ & $\begin{array}{l}\text { Permanente } \\
\text { (hasta que } \\
\text { Fuerza Loretana } \\
\text { siga en el Gorel) }\end{array}$ & Permanente & Permanente & $\begin{array}{l}\text { Esporádico } \\
\text { (solo en el } \\
\text { verano) }\end{array}$ & $\begin{array}{l}\text { Esporádico } \\
\text { (solo en el } \\
\text { verano) }\end{array}$ \\
\hline
\end{tabular}

Un factor que ayuda a los jóvenes indígenas a sentirse más confiados y a socializar es el hecho de juntarse entre ellos, sin importar el pueblo del cual provengan, ya que suelen tener las mismas necesidades. A.U.J. indicó que actualmente se siente parte de la organización, incluso fue vicepresidente. Sin embargo, en el pasado sintió exclusión de sus compañeros por provenir de Amazonas y no de Loreto, como la mayoría. Sus amigos lo apoyan emocionalmente y suele 
conversar con un par de compañeras que, al igual que él, no cuentan con muchos recursos económicos. G.M.T. señaló que su mayor problema como universitaria era relacionarse y comentó que la ayudaba a sentirse más tranquila el hecho de que en la Facultad de Educación tiene una compañera huitoto y una kichwa con las cuales se junta para hacer trabajos.

J.M.A. señaló que, durante los comienzos de la Oepiap, muchos compañeros tenían problemas académicos por la deficiencia de los sistemas educativos rurales y encontraron apoyo en instituciones políticas de izquierda:

[...] Nos encontramos con grupos políticos en la universidad, llámese de izquierda y los apristas [...]. Ellos en un momento se ofrecen para conseguirnos docentes que nos refuercen los cursos. Habían apoyado con dos, tres docentes; nos reforzábamos, pero nos jalaban para estar adentro de su línea política. Al ver esta situación, no nos enlazamos con las organizaciones [...]. Nuestra identidad y forma no concordaba (M. J., 27 de marzo de 2014).

El convenio establecía que la Ogebu debía coordinar con cada facultad para que estas designen tutores encargados de dar consejerías a los estudiantes indígenas, sin embargo, esto no ocurría. Otro incumplimiento del convenio se daba por la Aidesep, ya que la asociación se comprometió a «organizar por lo menos dos encuentros anuales de estudiantes indígenas y docentes de la UNAP, con expertos en Educación Intercultural Bilingüe en educación superior, para monitorear el proceso de formación profesional y académica de los estudiantes indígenas» (Aidesep-Gorel-UNAP, 2012, p. 4).

Acerca de los mayores retos, J.M.A. indicó que no dominaba perfectamente el castellano, lo cual era un problema cuando tenía que exponer en clases, por lo que trataba de asistir a charlas para mejorar. Además, hacía grupos de estudio con compañeros, y si bien solicitaba asesorías a los profesores, estos necesitaban un incentivo económico. A.U.J. también hacía grupos de estudio con algunos amigos del aula y sus amigos lo trataban de apoyar con información necesaria para los trabajos.

G.M.T. señaló que los profesores no brindaban asesorías y no estudiaba en grupo porque a veces sus compañeros no podían, pero solía preguntar a sus compañeros por el significado de algunas palabras y pedía ejemplos. Asimismo, P.M. le prestaba una laptop con acceso a internet para hacer sus investigaciones. D.C.P. señaló que utilizaba un libro para estudiar matemáticas que contiene ejercicios y técnicas de estudio. También pedía exámenes pasados y les sacaba copia. Él ha logrado tener éxito en algunos casos y se mostraba seguro de poder terminar su 
carrera en 2014, lo cual sucedió. Z.C.A. consideraba que debía poner especial empeño en los cursos de matemáticas y estadística y señaló que hacía ejercicios de matemáticas de manera individual. Sus mejores amigos también tenían dificultades y no conocía a los estudiantes con notas altas, por lo cual le querían cobrar.

Los estudiantes que persisten en culminar sus estudios universitarios gracias a la articulación de estrategias de distintos tipos desafían la estructura social de un contexto que promueve la inequidad, y enfrentan los distintos retos académicos, económicos y de socialización que pueden encontrar durante su vida universitaria.

\section{CONCLUSIONES}

Acostumbrarse a la ciudad y al ritmo universitario fue difícil para los jóvenes universitarios awajún, ya que el ingreso al espacio universitario implica una serie de retos relacionados con la socialización y el nivel académico. Sin embargo, se observó que la mayoría ya se estaba acostumbrando a su vida en Iquitos y a los retos que impone la universidad.

Los problemas de socialización se relacionan con el temor que tienen de ser discriminados por ser indígenas. Por otra parte, los estudiantes no indígenas y los profesores de los jóvenes awajún indicaron que muchas veces los mismos estudiantes indígenas se autoexcluían por temor al rechazo y preferían mantener un perfil bajo dentro del aula. Por el contrario, el perfil bajo era dejado de lado cuando los jóvenes indígenas luchaban por sus derechos o cuando se encontraban en frente de autoridades indígenas. En dichos espacios, la mayor parte se sentían empoderados y expresaban su identidad indígena. También es importante señalar que algunos compañeros y docentes valoraban los saberes prácticos de los jóvenes awajún, ya que resaltaban sus habilidades en las prácticas de campo y en cursos de supervivencia.

Los retos académicos son un problema latente causado por los desafíos característicos de la educación formal rural, frente al cual la UNAP no ofrece ningún programa de acompañamiento o curso propedéutico, lo cual está estipulado en el convenio interinstitucional. Sin embargo, la mayoría de los estudiantes awajún se sentían encaminados en la meta de volverse universitarios, ya que sus esfuerzos les permitían obtener resultados que se veían reflejados en buenas notas en los cursos. Si bien al comienzo los jóvenes sentían que acostumbrarse a la ciudad y al ritmo universitario era difícil; se estaban acostumbrando a su vida en Iquitos y a los retos académicos y de socialización que impone la universidad. 
Las condiciones bajo las cuales los estudiantes indígenas se encontraban en la UNAP han mejorado con el tiempo, ya que la Oepiap ha aliviado los retos económicos de los jóvenes a través de los años. Las primeras generaciones de estudiantes indígenas tenían retos más grandes como darse a conocer frente a las autoridades universitarias, visibilizarse y reclamar por derechos como la matrícula gratuita y el acceso al comedor universitario.

A pesar de que la situación de los jóvenes indígenas haya mejorado en términos generales con la ayuda de instituciones como el Gorel, los jóvenes awajún con los cuales se trabajó indicaron no encontrarse totalmente satisfechos con las condiciones en las que se encuentran tanto en Iquitos como en la UNAP, ya que muchas veces los recursos económicos eran escasos para realizar adecuadamente las labores estudiantiles. Se ha podido observar que las dificultades económicas y los retos académicos han sido los principales causantes de la deserción universitaria. En la actualidad, una de las estudiantes awajún abandonó la universidad para regresar a su pueblo. Sin embargo, en general la deserción universitaria ha disminuido gracias a las redes de apoyo construidas por Oepiap durante los últimos años.

La Oepiap es una plataforma de conexión con otros actores sociales como las autoridades de la UNAP, los Municipios o el Gorel. Por lo tanto, la institución les permite a los jóvenes awajún desarrollar un capital social. Esta es una estrategia colectiva para satisfacer las necesidades básicas que a su vez permite mantener la identidad social y cultural de sus integrantes por lograr que la adaptación a la cultura urbana sea más sencilla. Formar parte de la organización significa no tener que ingresar a un espacio totalmente desconocido y hostil para las actuales generaciones de jóvenes indígenas migrantes, ya que la organización tiene la función de guía en el difícil proceso de adaptación. Además, pertenecer a una organización que en el pasado se ha dado a conocer como un actor clave en la reivindicación de los jóvenes indígenas en Iquitos permite que los miembros de esta se sientan orgullosos de su identidad.

Por otro lado, la modalidad de ingreso de los pueblos indígenas en la UNAP es un tipo de acción afirmativa no intercultural, ya que se da un proceso unilateral en la cual los alumnos se deben acomodar a las normas y prácticas dominantes. La mayor parte de acciones afirmativas no buscan ser interculturales, solo multiculturales, y esto provoca que la educación superior peruana siga reproduciendo desigualdad en términos económicos, étnicos y sociales y que los jóvenes provenientes de grupos étnicos minoritarios sigan siendo invisibilizados por el Estado peruano. Las instituciones no se adaptan a los estudiantes indígenas ni a la 
diversidad del país, y esto se refleja en una estructura académico-administrativa y en planes de estudio estáticos, y en la indiferencia de muchas autoridades, profesores y alumnos.

El diseño de acciones afirmativas debería emplear un enfoque interculturaltransversalizador que asegure una interacción basada en el diálogo y respeto entre los distintos grupos; y un enfoque comunitario-empoderador en donde se piense la excelencia académica a partir de la valoración de las prácticas y saberes originarios de los distintos contextos.

La falta de cambios estructurales de carácter intercultural en universidades convencionales provoca que haya una serie de cuestionamientos acerca del proceso de profesionalización de los estudiantes indígenas: ¿Este proceso implica que los estudiantes adquieran los patrones de comportamiento de aquellos que algunas veces los excluyeron y que se vuelvan occidentales? ¿Qué trastornos ideológicos e identitarios implica el proceso de profesionalización? ¿Implica no retornar a sus comunidades de origen y estigmatizar a los suyos? Una posible solución al último cuestionamiento es que durante la carrera los estudiantes se vinculen con sus comunidades a través de la gestión de proyectos y la investigación-acción, lo cual sería posible con un modelo de gestión de la educación participativo y descentralizado.

Es crucial trabajar con diferentes actores, en especial con las distintas comunidades de donde provienen los estudiantes para que supervisen y asesoren las acciones afirmativas con el fin de que los procesos relacionados con la construcción curricular provengan desde saberes tradicionales y locales distintos del saber occidental. Asimismo, se considera importante que dichos actores y las asociaciones indígenas trabajen en conjunto con la Ogebu de UNAP con el fin de garantizar el bienestar de los estudiantes provenientes de distintas realidades como los jóvenes awajún.

La promoción de la interculturalidad en las acciones afirmativas es clave, ya que los alumnos a los cuales se dirigen provienen de contextos culturales muy diversos; por lo tanto, la interacción, diálogo y respeto entre distintos grupos culturales es fundamental para su bienestar, no solo la convivencia que actualmente se da en algunas universidades como la UNAP. 


\section{REFERENCIAS BIBLIOGRÁFICAS}

Aidesep, Gorel y UNAP (2012). Convenio de Cooperación Interinstitucional entre el Gobierno Regional de Loreto, la Universidad Nacional de la Amazonía Peruana y la Asociación Interétnica de Desarrollo de la Selva Peruana.

Buu-Sao, Doris (2011). Le gouvernement des contestaires l'Amazonie péruvienne: le cas de l'organisation des étudiants indigènes OEPIAP. Mémoire: IEP.

Castro, Juan Francisco y Gustavo Yamada (2011). Brechas étnicas y de sexo en el acceso a la educación básica y superior en el Perú. Lima: CIUP.

Cavero, O. (2011). Después del baguazo: informes, diálogo y debates. Cuaderno de trabajo, 13 (Serie Justicia y Conflictos 1). Lima: Departamento de Ciencias Sociales Pontifica Universidad Católica del Perú.

Cuenca, Ricardo (ed.) (2012). Educación superior: movilidad social e identidad. Lima: IEP.

Cunningham, Mirna (2008). Educación indígena, interculturalidad y desarrollo autónomo. Nicaragua: CADPI.

Degregori, Carlos Iván (1986). Del mito de Inkarri al mito del progreso: poblaciones andinas, cultura e identidad nacional. Socialismo y Participación, 36, 49-56.

Dietz, Gunther (2009). Los actores indígenas ante la «interculturalización» de la educación superior en México: ¿empoderamiento o neoindigenismo? Revista Latinoamericana de Educación Inclusiva, 3(2), 55-75.

Espinosa de Rivero, Óscar (2007). Para vivir mejor: Los indígenas amazónicos y su acceso a la educación superior en el Perú. ISEES - Inclusión Social y Equidad en la Educación Superior, 2, 85-116.

Espinosa de Rivero, Óscar (2012). To Be Shipibo Nowadays: The Shipibo-Konibo Youth Organizations as a Strategy for Dealing with Cultural Change in the Peruvian Amazon Region. The Journal of Latin American and Caribbean Anthropology, 17(3), 451-471. https://doi.org/10.1111/j.1935-4940.2012.01252.x

Instituto Nacional de Estadística e Informática (INEI) (2016). Encuesta Nacional de Hogares (Enaho).

Mato, Daniel (2007). Interculturalidad y educación superior: diversidad de contextos, actores, visiones y propuestas. Nómadas, 27, 62-73. Colombia: Universidad Central.

Omar, Alicia (2006). Las perspectivas de futuro y sus vinculaciones con el bienestar subjetivo y la resiliencia en adolescentes. En Martina Casullo (ed.), Psicodebate 7: Psicología, Cultura y Sociedad (pp. 141-154). Buenos Aires, Universidad de Palermo. 
Organización de Estudiantes de los Pueblos Indígenas de la Amazonía Peruana (OEPIAP). (2011). Waymaku, 1. Iquitos.

Pons, Gonzalo (2013). Bienestar subjetivo en la Amazonía peruana: la estructura de sus necesidades. Tesis de licenciatura en Letras y Ciencias Humanas con mención en Psicología Social. Pontificia Universidad Católica del Perú, Facultad de Letras y Ciencias Humanas.

Sanborn, Cinthya y Alonso Arrieta (2012). Universidad y acción afirmativa: balance y agenda pendiente. En Cinthya Sanbor (ed.), La discriminación en el Perú: balance y desafios. Lima: Universidad del Pacífico.

Sarfaty, Sara (2011). La experiencia de ser estudiante universitario e indígena amazónico: prácticas discriminatorias al interior de la Universidad Nacional Intercultural de la Amazonía (UNIA). Tesis de licenciatura en Ciencias Sociales con mención en Antropología. Lima: Pontifica Universidad Católica del Perú, Facultad de Ciencias Sociales.

Schmelkes, Sylvia (2004). La educación intercultural: un campo en proceso de consolidación. Revista Mexicana de Investigación Educativa, 9(20), 9-13. México D.F.: COMIE.

Trapnell, Lucy (2008). La experiencia del Programa de Formación de Maestros Bilingües de la Amazonía Peruana. En Daniel Mato (ed.), Diversidad cultural e interculturalidad en educación superior. Experiencias en América Latina (pp. 403-412). Caracas: IESALC-Unesco.

\section{SIGLAS Y ACRÓNIMOS UTILIZADOS}

$\begin{array}{ll}\text { Aidesep } & \text { Asociación Interétnica de Desarrollo de la Selva Peruana } \\ \text { EIB } & \text { Educación Intercultural Bilingüe } \\ \text { Enaho } & \text { Encuesta Nacional de Hogares } \\ \text { Formabiap } & \text { Formación de Maestros Bilingües de la Amazonía Peruana } \\ \text { Gorel } & \text { Gobierno Regional de Loreto } \\ \text { Oepiap } & \text { Organización de Estudiantes de los Pueblos Indígenas de la Amazonía } \\ & \text { Peruana } \\ \text { Ogebu } & \text { Oficina General de Bienestar Universitario } \\ \text { Orpio } & \text { Organización Regional de los Pueblos Indígenas del Oriente } \\ \text { RAL } & \text { Red Ambiental Loretana } \\ \text { UNAP } & \text { Universidad Nacional de la Amazonía Peruana } \\ \text { UNIA } & \text { Universidad Nacional Intercultural de la Amazonía } \\ \text { UNMSM } & \text { Universidad Nacional Mayor de San Marcos } \\ \text { UNSAAC } & \text { Universidad Nacional de San Antonio Abad del Cusco } \\ \text { UVI } & \text { Universidad Veracruzana Intercultural }\end{array}$

